\title{
Neurofeedback effects on EEG connectivity in children with reading disorder: $I$. Coherence
}

\author{
Lucero Albarrán-Cárdenas ${ }^{1}$, Juan Silva-Pereyra ${ }^{2}$, Benito J. Martínez-Briones ${ }^{1}$, Jorge Bosch-Bayard ${ }^{3}$, and Thalía Fer- \\ nández ${ }^{1, *}$
}

1 Departamento de Neurobiología Conductual y Cognitiva, Instituto de Neurobiología, Universidad Nacional Autónoma de México Campus Juriquilla, Querétaro QE 76230, Mexico; albarranluc@hotmail.com (L.A.-C.); benjavik332@gmail.com (B.J.M.-B.); thaliafh@yahoo.com.mx (T.F.)

2 Facultad de Estudios Superiores Iztacala, Universidad Nacional Autónoma de México, Tlanepantla, Estado de México MX 54090, Mexico; jsilvapereyra@gmail.com (J. S.-P.)

3 McGill Centre for Integrative Neuroscience (MCIN), Ludmer Centre for Neuroinformatics and Mental Health, Montreal Neurological Institute (MNI), McGill University, Montreal, QC H3A 2B4, Canada; oldgandalf@gmail.com (J.B.-B.)

* Correspondence: thaliafh@yahoo.com.mx

\begin{abstract}
Electroencephalograms (EEGs) of children with reading disorder (RD) are characterized by higher power and coherence in slow frequencies (delta and theta bands) and lower power and coherence in the alpha band compared to EEGs of typically developed children. Neurofeedback (NFB) is useful for treating learning disorders by reinforcing the reduction of the theta/alpha ratio. This efficacy is supported by EEG power normalization and cognitive-behavioral improvement. To further explore brain changes in isolated areas, this study aimed to explore the effects of this NFB protocol on functional connectivity (coherence) in children with RD. Twenty children with RD and an abnormally high theta/alpha ratio underwent 30 sessions of NFB. After treatment, $90 \%$ of participants learned to decrease their theta/alpha ratio, and on average, children increased their reading accuracy and comprehension scores. Interhemispheric coherence diminished in the delta, theta and beta bands, mainly between frontopolar regions. Intrahemispheric coherence decreased in delta, theta and beta bands and increased in alpha band. The reduction in theta coherence between the left frontal area and other regions of the left hemisphere had particular relevance for reading. These results suggest that this NFB treatment could positively impact reading-related functions in the brain networks of children with RD.
\end{abstract}

Keywords: connectivity; coherence; neurofeedback; reading disorder; learning disorder; dyslexia

\section{Introduction}

Learning to read is a cognitive process that takes place at an early age; it is a process that requires formal training and depends on the development and coordination of multiple brain processes in different regions that participate in its acquisition [1-3].

Although most people learn to read easily, $5-17 \%$ of school-age children $[4,5]$ have difficulties acquiring reading skills [6]. When these difficulties manifest along with low scores on standardized reading tests (1.5 standard deviations below the population mean for the child's age), they provide diagnostic certainty of the specific learning disorder with impairment in reading (RD), which is considered specific because it is not attributable to intellectual disability, hearing or vision disorders, or neurological or motor disorders [7].

Reading problems have been reported to be associated with structural and/or functional abnormalities, specifically in left perisylvian regions [4]. Numerous noninvasive techniques can provide us with information on brain function. Due to its high temporal resolution, electroencephalogram (EEG) recordings provide a useful tool for the study of brain dynamics [8-11] and for estimating connectivity [12].

Previous studies have reported that children with learning disabilities have a different maturational process of EEG coherence than children with typical development 
[13]. Although there is no consensus yet about what characterizes the resting EEG of children with RD, the most reported EEG pattern is an excess of slow activity, mainly in the theta frequency range [14-17], and an alpha activity deficit [18-20] when compared to children with typical development. This EEG pattern corresponds to a younger child; therefore, this finding has been interpreted as these children with RD exhibiting a delay in electroencephalographic maturation [15]. Most of the studies exploring brain activity in children with learning disabilities using EEGs have been based on EEG power analysis, and few have focused on functional connectivity measures.

Studying brain connectivity not only allows us to identify isolated brain areas that participate in a certain function but also shows us the neural networks involved and their interaction [21]. EEG coherence has been widely used to study the functional connectivity between different brain areas [22] because it provides us with a quantifiable degree of functional connectivity that exists between the structures underlying the recording electrodes at the scalp [23-26]. Currently, there are better measures to assess EEG connectivity [27]; however, we selected the coherence between sensors because it is the measure used in the clinical setting. Furthermore, having been used previously to characterize the EEG of RD children, it is possible to compare our results with previous results and validate its reproducibility.

Poor literacy performance has been associated with higher coherence values, specifically in the delta, theta, and beta frequency bands [28, 29], and lower coherence values in the alpha band $[29,30]$. This lower alpha coherence was replicated in adults with dyslexia [31]. In general, there was a tendency of increasing coherence values in development except in the theta band [32]. When attention is brought to interhemispheric and intrahemispheric coherence, children with dyslexia are characterized by lower interhemispheric coherence and higher intrahemispheric coherence compared to the coherence of their classmates of the same age $[33,34]$.

A treatment aiming to reduce theta activity and increase alpha activity in children with learning disorders who also have delayed electroencephalographic maturation could be useful as it could normalize their EEG characteristics and concomitantly improve their reading performance. A neurofeedback (NFB) treatment that positively reinforces the reduction of the theta/alpha ratio has been shown to be effective in reducing this ratio, reorganizing the EEG, and improving cognitive-behavioral performance [35-37]; treatment effects last for at least two years [38]. In these prior studies, changes in the absolute power (AP) and relative power (RP) of EEG signals were explored. However, currently, a more realistic perspective in cerebral function research has been provided by neural networks and connectivity and not just by the identification of isolated areas that show changes in neuronal activation [39]. In children with RD effectively treated with this NFB protocol, it would be interesting to explore whether this treatment has had a positive effect on electroencephalographic connectivity between brain areas related to the reading process.

We aimed to explore the effects of NFB protocol that reinforces the reduction of the theta/alpha ratio on functional connectivity in order to evaluate if this treatment have the capability to normalize the altered mechanisms that underlie reading in RD children with EEG maturational lag. Based on the coherence of control children vs. RD children and from studies of NFB in children with a reading disorder, we hypothesized that NFB would produce a reorganization that would consist of a) a reduction in intrahemispheric delta and theta coherence, b) an increase in intrahemispheric alpha coherence and c) an increase in interhemispheric coherence.

\section{Materials and Methods}

The Ethics Committee of the Institute of Neurobiology of the National Autonomous University of México approved the experimental protocol INEU/SA/CB/146 on 1 July 2015, which followed the Ethical Principles for Medical Research on Human Subjects established by the Declaration of Helsinki [40]. 


\subsection{Participants}

Twenty right-handed subjects aged 7 to 11 years were included in this study. The participants were volunteers and were referred by social workers, teachers, and parents from different public and private schools in Querétaro, México. All participants in the sample met the diagnostic criteria for RD according to the Diagnostic and Statistical Manual of Mental Disorders 5th Edition. These criteria were as follows: a) The child showed persistent difficulties in acquiring and/or using academic skills related to reading (accuracy, comprehension and/or speed). Reading skills were measured with the Child Neuropsychological Assessment-2 [41]; children included in the study had percentile scores $<9$ in at least one of the subscales of the reading domain of the ENI-2. b) Reading difficulties began in the first years of schooling, and not because the participants had intellectual disabilities; the intelligence quotient (IQ) of the participants was measured with the Wechsler Intelligence Scale for Children 4th edition [42]. The IQ scores obtained by each child of the sample were greater than or equal to 80. c) The children have not hearing impairment; five children used lenses to correct their visual difficulties. Any children presented neurological or psychiatric pathology, except by the learning disorder. A neuro-pediatric examination was performed, and the Mini-International Neuropsychiatric Interview for Children and Adolescents (MINI Kid) [43, 44] was used; this assessment was chosen to determine that the subjects did not have any disorder other than the RD. Although children had attentional disabilities, these were not enough to establish an Attention Deficit Hyperactivity Disorder (ADHD) diagnostic. No children were medicated. d) No severe socioeconomic or cultural problems were evident [45]. All participants had an abnormally high EEG theta/alpha ratio compared to a normative database [46, 47]. All children and their parents signed informed consent forms.

\subsection{EEG Recording and Analysis}

The electroencephalogram was recorded in a resting condition with eyes closed from the 19 leads of the international 10-20 system (Fp1, Fp2, F3, F4, C3, C4, P3, P4, O1, O2, F7, F8, T3, T4, T5, T6, Fz, Cz, and Pz), referenced to the linked earlobes (A1A2). For this purpose, Medicid ${ }^{\mathrm{TM}}$ IV equipment (Neuronic Mexicana, SA, Mexico City, Mexico) and a v5.0 Track Walker ${ }^{\mathrm{TM}}$ recording system were used. The amplifier bandwidth was set between 0.5 and $50 \mathrm{~Hz}$. All electrode impedances were equal to or less than $5 \mathrm{k} \Omega$, and the signal was amplified with a gain of 20,000. EEG data were sampled every $5 \mathrm{~ms}$ and edited offline. Twenty-four artifact-free segments of 2.56 seconds were selected. EEG analysis was performed offline. The fast Fourier transform was applied to the data and the cross-spectral matrices were obtained every $0.39 \mathrm{~Hz}$ to calculate the AP and coherence values of each of the bands (delta: $0.5-3.5 \mathrm{~Hz}$, theta: $3.6-7.5 \mathrm{~Hz}$, alpha: 7.6-12.5 Hz, and beta: $12.6-19 \mathrm{~Hz})$.

\subsection{Z-values for the theta/alpha ratio}

To calculate z-values for the theta/alpha ratio, AP in theta and alpha bands was computed for each electrode, and the geometric power [48] was subtracted from the cross-spectral matrix.

The log value (theta AP/alpha AP) was computed, and z-values for this logarithm were calculated using the equation:

$$
\mathrm{Z}=[\text { thetaAP/alphaAP }-\mu] / \sigma
$$

where $\mu$ and $\sigma$ are the mean value and the standard deviation, respectively, of a normative sample that is the same age as the subject [46, 47]. Considering that the EEG of this population is characterized by having more theta activity and less alpha activity than children with typical development, having a z-value greater than 1.645 (1-tailed distribution, $\mathrm{p}=0.05$ ) in at least one lead was also designated as an inclusion criterion. Treatment was delivered via the lead with the highest abnormal z-value.

\subsection{NFB treatment}


Treatment was performed using an NFB program adapted for the Medicid IV registration system. A threshold level was selected in which the subject obtained a reward between 60 and $80 \%$ of the time. The stimulus used as a reward was a tone of $500 \mathrm{~Hz}$ at 60 $\mathrm{dB}$. Every 3 minutes, the threshold level was updated based on the subject's performance. Each subject received 30 training sessions three times a week, and the duration of each session was 30 minutes.

\subsection{Posttreatment analysis}

The Wechsler Intelligence Scale for Children 4th edition and the ENI-2 Child Neuropsychological Assessment were applied before NFB treatment for diagnostic purposes and after treatment to assess the cognitive effects of the treatment. It was necessary for a year to elapse between the first and second application of these tests; therefore, second application was applied two months after treatment. The application manual of these tests stipulates that a year must pass between the first and second applications. Since recruitment lasted several months and the treatment period included the same dates for all children, this timeline fulfilled the scheduling requirements.

In a session following the tests' application, the posttreatment EEG was recorded with two purposes: a) to assess whether NFB-induced learning occurred and b) to evaluate the NFB effects on coherence. We considered that a child learned if his/her z-value decreased.

\subsection{Coherence analysis}

EEG data were exported from Medicid IV to calculate coherence with Neuroguide 2.6.5 software. Intrahemispheric coherence was obtained considering all leads except the midline (Fp1, F3, C3, P3, O1, F7, T3, and T5 in the left hemisphere and Fp2, F4, C4, P4, O2, F8, T4, and T6 in the right hemisphere), and interhemispheric coherence was obtained considering only homologous derivations. EEG coherence values were calculated before and after treatment.

\subsection{Statistical analysis}

A series of permutation analyses was performed to compare the data before and after treatment using Student's t-test for dependent samples. Independent analyses were performed for the variables total IQ, reading precision, reading comprehension, reading speed, theta/alpha ratio, interhemispheric coherence, and intrahemispheric coherence.

\section{Results}

\subsection{NFB-induced learning}

In Figure 1, it can be observed that the theta/alpha ratio of the most abnormal derivation for everyone decreased significantly after treatment $(t=4.4497, p=0.0004)$. Fifty-five percent of the treated children normalized their theta/alpha ratio values in the derivation used to give NFB. However, $90 \%$ of the participants decreased their theta/alpha ratio even though they had not reached normal values. 


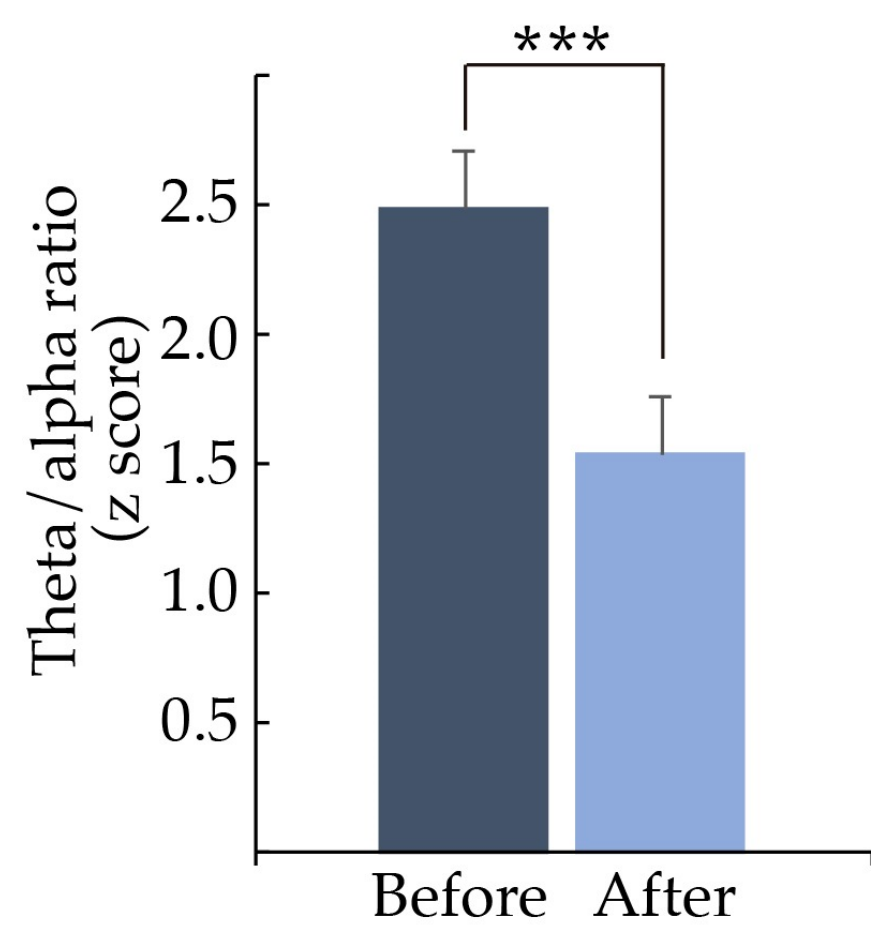

Figure 1. Mean and standard deviation of the theta/alpha ratio before and after NFB treatment. The Z-score of the theta/alpha ratio decreased significantly after NFB treatment.

\subsection{Behavioral outcomes}

There were no significant changes in total IQ after treatment. In the reading variables included in the infant neuropsychological evaluation, significant improvement was observed in precision $(t=2.1391, p=0.0194)$ and comprehension $(t=-3.4554, p=0.0010)$, as shown in Figure 2; no significant result was found in speed reading when comparing results before vs. after NFB.

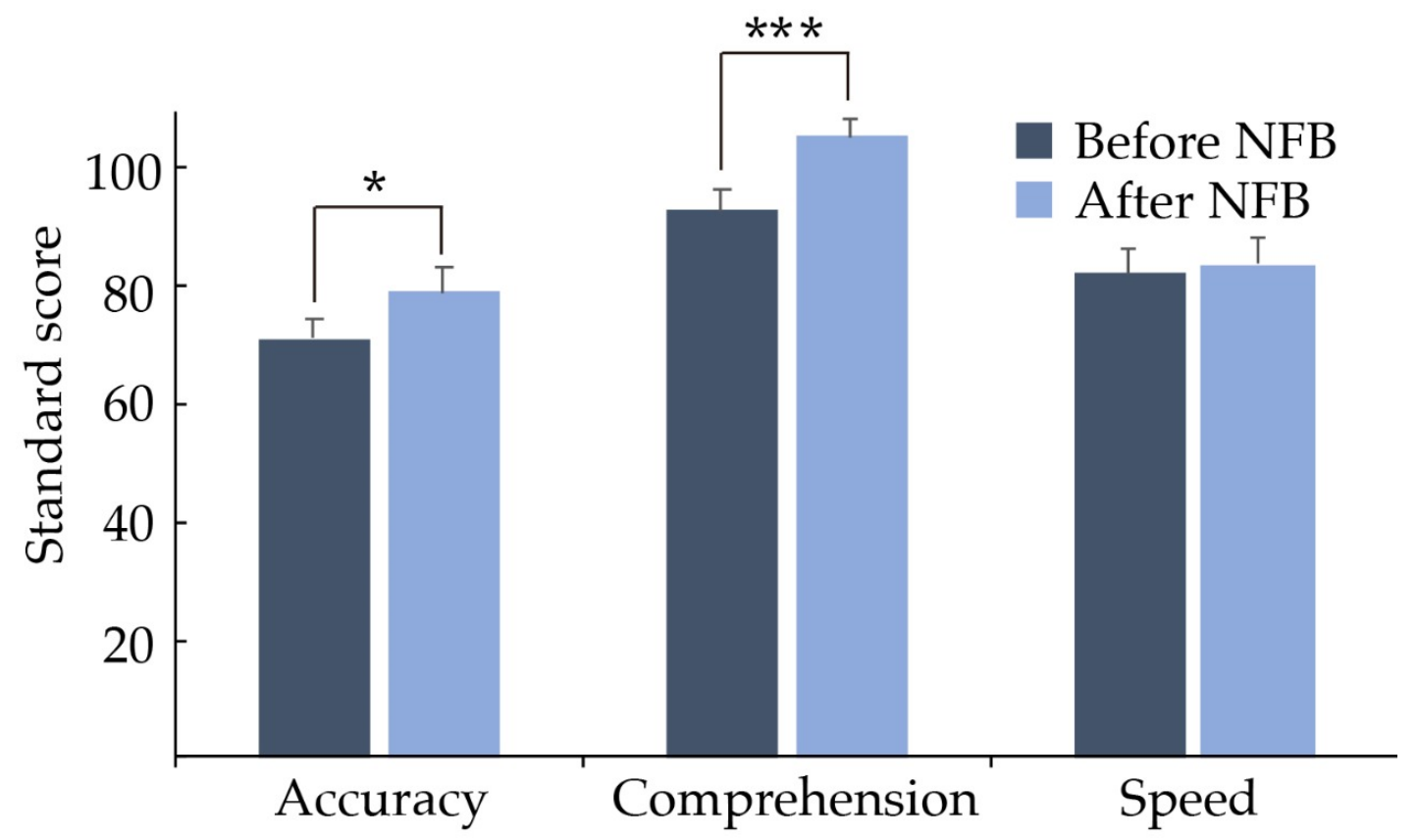

Figure 2. Mean and standard deviation of the Child Neuropsychological Assessment-2 in the reading domain before (dark blue) and after (light blue) NFB treatment. Reading accuracy and comprehension increased significantly $\left({ }^{*} \mathrm{p}=0.01,{ }^{* *} \mathrm{p}=0.01\right)$. 


\subsection{Functional connectivity changes}

\subsubsection{Intrahemispheric coherence}

In Figure 3, the results of intrahemispheric coherence in both hemispheres can be observed. In the left hemisphere, P3-O1 coherence in the alpha frequency band increased significantly $(t=-3.2083, p=0.0342)$. In the theta frequency band, the coherence values decreased significantly in F3-P3 $(t=3.6818, p=0.0144)$, F3-01 $(t=3.3866, p=0.0294)$, F3-T3 $(\mathrm{t}=3.8031, \mathrm{p}=0.0112)$, F3-T5 $(\mathrm{t}=3.8038, \mathrm{p}=0.0112)$ and C3-P3 $(\mathrm{t}=4.9384, \mathrm{p}=0.0022)$. In the delta and beta bands, no significant changes occurred.

In the right hemisphere, beta coherence decreased in Fp2-F4 $(t=3.0484, p=0.0440)$, FP2-F8 $(t=3.1789, \mathrm{p}=0.0328)$ and P4-T4 $(\mathrm{t}=3.1180, \mathrm{p}=0.0384)$. In the theta band, the coherence values decreased in FP2-F8 $(t=3.1543, \mathrm{p}=0.0398)$ and F4-C4 $(\mathrm{t}=3.7558, \mathrm{p}=$ $0.0110)$. In the delta band, $C 4-T 4$ coherence decreased $(t=3.2628, p=0.0366)$. In the alpha band there were no significant changes.
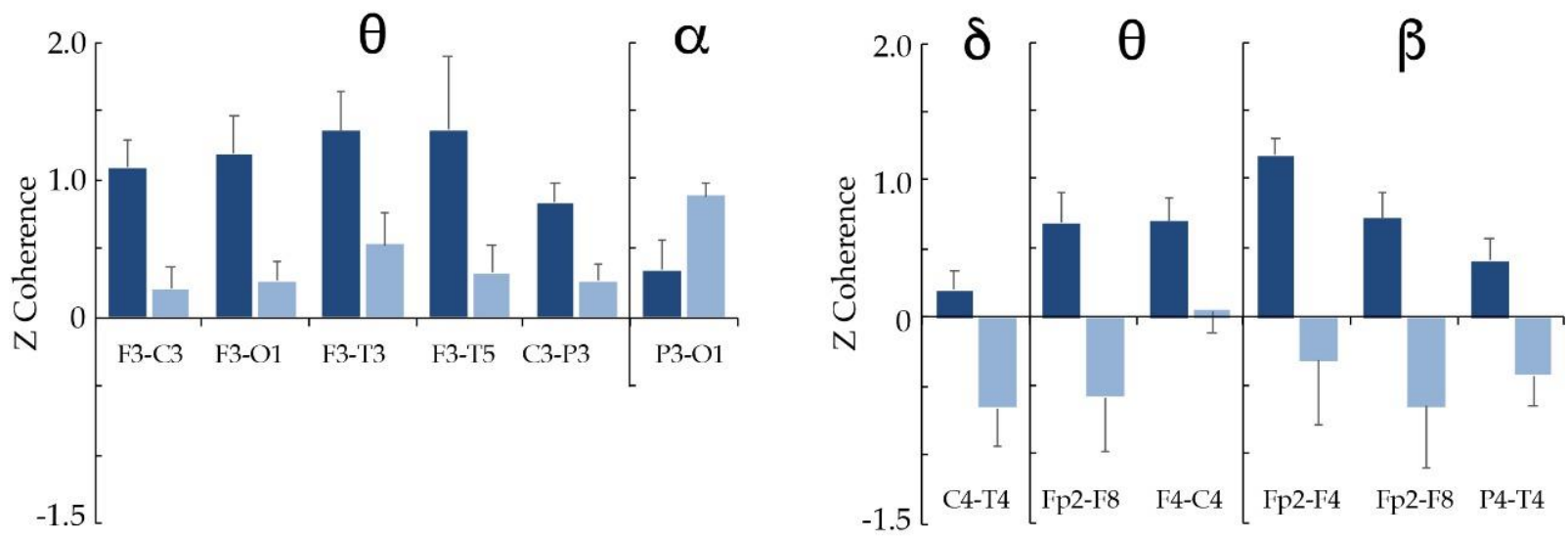

Left

Right

\section{Before NFB $\square$ After NFB}
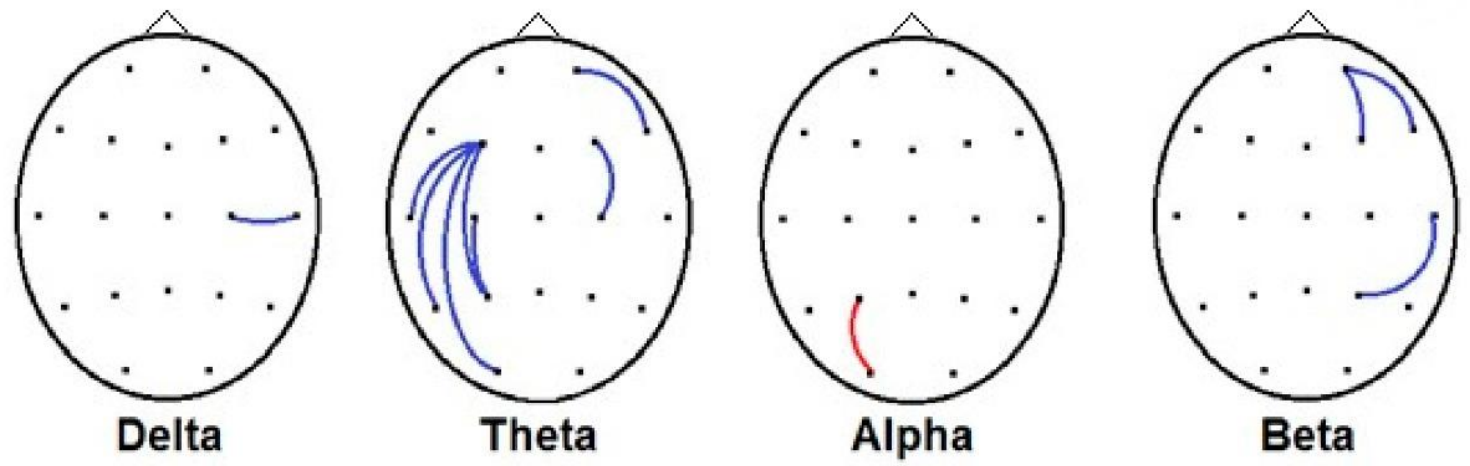

\section{Decreased coherence}

\section{Increased coherence}

Figure 3. Top: Bar graphs showing the significant changes in coherence $z$-values by frequency band (indicated at the top) when comparing intrahemispheric connectivity Before vs. After NFB treatment; left or right hemisphere is indicated below the graph. Bottom: Topographic representation of significant changes in z-coherence after NFB treatment, showing the increase or decrease in red or blue, respectively.

3.3.2. Interhemispheric coherence (homologous pairs) 
Figure 4 shows the changes in interhemispheric coherence between homologous pairs. In all bands, coherence values between frontopolar regions decreased, except in the alpha band, where there were no changes (delta Fp1-Fp2: $t=2.6653, p=0.0370$; theta Fp1-Fp2: $t=2.7395, p=0.0398$; beta Fp1-Fp2: $t=2.7592, p=0.0322$ ). In addition, coherence between posterior temporal (T5-T6: $\mathrm{t}=3.3042, \mathrm{p}=0.0148)$ and parietal regions (P3-P4: $\mathrm{t}=$ 3.1918, $\mathrm{p}=0.0176$ ) decreased in the theta band.

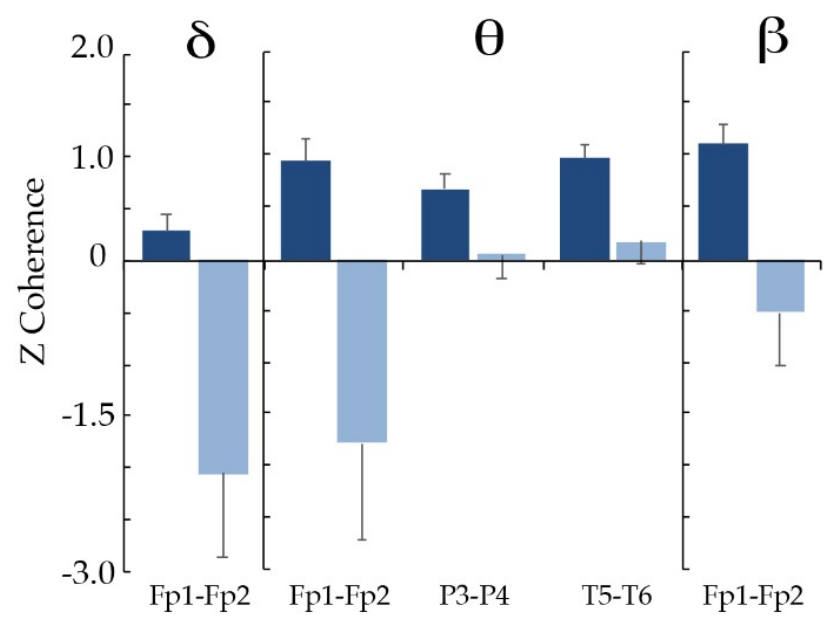

Before NFB

After NFB
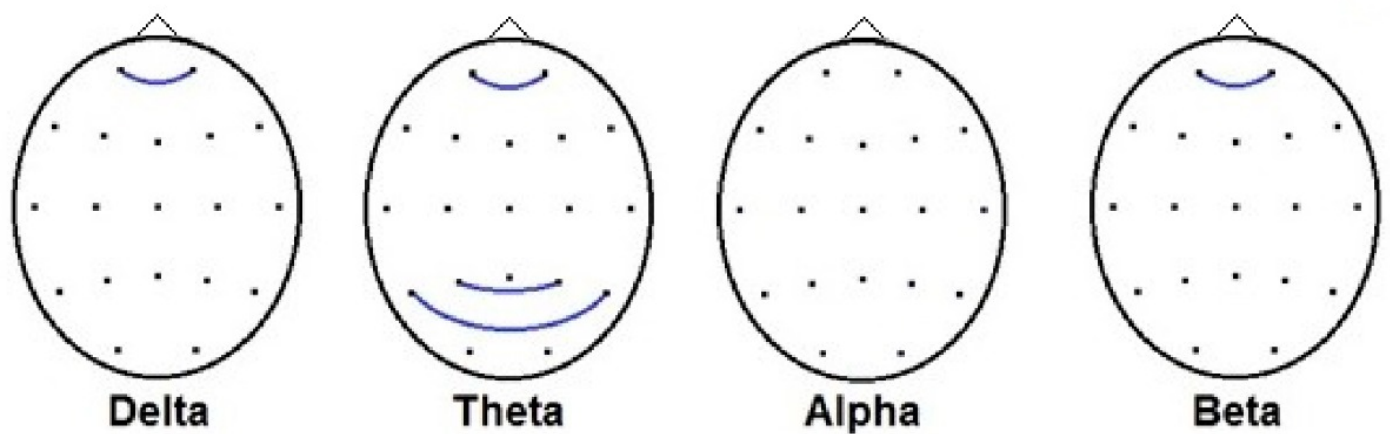

Decreased coherence

Figure 4. Significant differences in interhemispheric coherence between homologous pairs when before and after treatment were compared. Top: Bar graph showing the significant changes in coherence z-values by frequency band (indicated at the superior part). Bottom: Topographic representation of significant changes at the scalp; blue lines indicate that coherence was reduced after treatment.

\section{Discussion}

Our objective in this study was to explore the changes in EEG coherence patterns in children with RD treated with NFB using a protocol that reinforces the reduction in the theta/alpha ratio. Given that this protocol has already demonstrated its efficacy when treating the condition of this population [35-38], no control group was used in this study.

Previous studies have reported that NFB-induced learning occurs in approximately $80 \%$ of treated individuals [36]. In our study, a significant reduction in the theta/alpha 
ratio was demonstrated in the lead used to guide NFB. Ninety percent of the participants reduced this quotient, and 55\% reached normalization.

It was expected that as a consequence of NFB treatment, a reduction in intrahemispheric delta and theta coherence, an increase in intrahemispheric alpha coherence, and an increase in global interhemispheric coherence would be observed. Our results confirm the hypotheses regarding intrahemispheric coherence. We found a reduction in delta coherence between central and right temporal regions and in theta coherence, between left frontal regions and other regions of the same hemisphere and between frontal regions of the right hemisphere. An increase in alpha coherence was also observed between the parietal and occipital regions of the left hemisphere. In addition to this hypothesis, we found a reduction in beta coherence between regions of the right hemisphere with frontal dominance.

However, the hypothesis regarding interhemispheric coherence was wholly rejected by our results. We found a reduction in coherence between frontopolar regions in all bands except for the alpha band and a reduction in theta coherence between posterior parietal and temporal regions.

In studies conducted in which EEG coherence in the pediatric population was analyzed, the tendency has been to associate high values of coherence, mainly in the delta and theta bands, with cognitive dysfunction [49]. For example, Thatcher [50] reported high coherence in children with low IQ scores. Chabot et al. [51], Barry et al. [52], and Clarke et al. [53] reported the same results in patients with ADHD. However, in studies conducted in children with learning disabilities, the results were not very consistent; this may be because children with learning disabilities are a heterogeneous population [54] and because they frequently have many comorbidities that have not been controlled in other studies.

In individuals with reading disorders, abnormalities in cortical connectivity have been found. These could be due to alterations in neuronal migration [31] reported by Galaburda et al. [55], who evidenced cortical anomalies in the brains of people with dyslexia in postmortem studies, which consisted of neuronal ectopias and dysplasias located mainly in the left perisylvian regions. Years later, using imaging techniques such as magnetic resonance imaging, the existence of a cortical network in the left hemisphere was reported that involves three specialized regions that participate in reading: 1) the left dorsal temporoparietal circuit located around the area of Wernicke, which specializes in phonological functions; 2) the left ventral occipito-temporal circuit that houses the visual area of word form, to which visual and orthographic recognition based on memory is attributed; and 3) the lower left frontal circuit classically called Broca's area that intervenes in the articulation and gesticulation of words [56].

In our study, a reduction in theta coherence was observed in areas of the left hemisphere that are spatially correlated with this previously described cortical network. However, as previously mentioned, EEG recordings are characterized by great temporal resolution, but they do not have good spatial resolution; therefore, we could not guarantee that it corresponds precisely to these structures directly involved in the reading process. Future studies would be necessary to perform analyses using other methods that could result in a more specific topographic localization.

Reading disorders have been associated with higher coherence values in the delta, theta, and beta bands [28, 29] and lower coherence values in the alpha band [29, 30]. Therefore, our results indicate a trend towards normalization of coherence patterns in treated children. This coherence maturation can be associated with improved reading comprehension and precision scores on the ENI-2 neuropsychological test since it occurs among regions involved in the previously described network.

To conclude, we consider that the identification and early diagnosis of RD allow specific and timely interventions to be carried out to reduce the impact of this disorder on a child's personal, school, and social life as much as possible. It also allows us to implement techniques that have already proven to be effective, such as NFB, which was confirmed in this study to contribute positively to the normalization of the function of the 
network involved in the reading process and, consequently, in children's academic performance.

Author Contributions: Conceptualization, L.A-C. and T.F.; methodology, L.A-C, J.S-P., B.M-B, J.B-B., T.F.; software, J.B-B.; formal analysis, L.A-C, J.S-P., T.F.; investigation, L.A-C, J.S-P., B.M-B, J.B-B., T.F.; resources, L.A-C, B.M-B, T.F.; data curation, L.A-C, J.S-P., B.M-B, J.B-B., T.F.; writing-original draft preparation, L.A-C.; writing-review and editing, L.A-C, J.S-P., B.M-B, J.B-B., T.F.; visualization, L.A-C, J.S-P.; supervision, J.S-P., J.B-B., T.F.; project administration, T.F.; funding acquisition, T.F. All authors have read and agreed to the published version of the manuscript.

Funding: This research was supported by CONACYT under the grant CB-2015-1-251309, and by the grants IN204613, IN205520, and IN207520 from Programa de Apoyo a Proyectos de Investigación e Innovación Tecnológica (PAPITT), DGAPA-UNAM, Mexico. Jorge Bosch-Bayard was supported by Brain Canada (243030), the Fonds de recherche du Québec (FRQ) HBHL FRQ/CCC Axix (246117), the CFREF/HBHL HIBALL, and Helmholtz (252428). During the realization of this work, Lucero Albarrán-Cárdenas (scholarship recipient: 626189) was a beneficiary of the CONACYT scholarship 473496.

Institutional Review Board Statement: The study was conducted according to the guidelines of the Declaration of Helsinki, and approved by the Ethics Committee of Instituto de Neurobiología, Universidad Nacional Autónoma de México (INEU/SA/CB/146; July 1st, 2015).

Informed Consent Statement: Informed consent was obtained from all children involved in the study, and their parents.

Data Availability Statement: Data will be uploaded to figshare-url

Acknowledgments: The authors are grateful for the children's and parents' cooperation in this study. The authors also acknowledge Fabiola García Martínez, Milene Roca-Stappung, Sonia Y. Cárdenas, Rodrigo Flores Gallegos, Paulina Rodríguez Leis, Bertha Elena Barrera Díaz, María Elena Juárez, Manuel Hinojosa Rodríguez, Héctor Belmont, Saulo Hernández, Nuri Aranda, Leonor Casanova, Carlos Sair Flores Bautista, Teresa Álvarez, Eduardo González-Moreira, Juan José Ortiz Retana, Bertha Esquivel Quiroz, Marco Olguín Araujo, Joel Bernardino Peláez, and Lourdes Lara for their technical assistance. This work was supported by the Programa de Apoyo a Proyectos de Investigación e Innovación Tecnológica (IN204613, IN205520, and IN207520) and the Consejo Nacional de Ciencia y Tecnología (CONACYT; CB-2015-01-251309). Lucero Albarrán is a beneficiary of a CONACYT scholarship (No. 473496).

Conflicts of Interest: The authors declare no conflict of interest.

\section{References}

[1] Galaburda, A.; Camposano, S. Dislexia Evolutiva: Un Modelo Exitoso de Neuropsicología Genética. Rev. Chil. Neuropsicol., 2006, 1 (1).

[2] Puente, A.; Jiménez, V.; Ardila, A. Anormalidades Cerebrales En Sujetos Disléxicos. Rev. Latinoam. Psicol., 2009, $41(1)$.

[3] Žarić, G.; Correia, J. M.; Fraga González, G.; Tijms, J.; van der Molen, M. W.; Blomert, L.; Bonte, M. Altered Patterns of Directed Connectivity within the Reading Network of Dyslexic Children and Their Relation to Reading Dysfluency. Dev. Cogn. Neurosci., 2017, 23. https://doi.org/10.1016/j.dcn.2016.11.003.

[4] Cutting, L. E.; Clements-Stephens, A.; Pugh, K. R.; Burns, S.; Cao, A.; Pekar, J. J.; Davis, N.; Rimrodt, S. L. Not All Reading Disabilities Are Dyslexia: Distinct Neurobiology of Specific Comprehension Deficits. Brain Connect., 2013, 3 (2). https://doi.org/10.1089/brain.2012.0116.

[5] Gabrieli, J. D. E. Dyslexia: A New Synergy between Education and Cognitive Neuroscience. Science. 2009. 
https://doi.org/10.1126/science.1171999.

[6] Démonet, J.-F.; Taylor, M. J.; Chaix, Y. Developmental Dyslexia. Lancet, 2004, 363 (9419), 1451-1460. https://doi.org/10.1016/S0140-6736(04)16106-0.

[7] American Psychiatric Association. Diagnostic and Statistical Manual of Mental Disorders; 2013. https://doi.org/10.1176/appi.books.9780890425596.744053.

[8] Hanlon, H. W.; Thatcher, R. W.; Cline, M. J. Gender Differences in the Development of EEG Coherence in Normal Children. Dev. Neuropsychol., 1999, 16 (3). https://doi.org/10.1207/S15326942DN1603_27.

[9] Haufe, S.; Nikulin, V. V.; Müller, K. R.; Nolte, G. A Critical Assessment of Connectivity Measures for EEG Data: A Simulation Study. Neuroimage, 2013, 64 (1). https://doi.org/10.1016/j.neuroimage.2012.09.036.

[10] Marzetti, L.; Del Gratta, C.; Nolte, G. Understanding Brain Connectivity from EEG Data by Identifying Systems Composed of Interacting Sources. Neuroimage, 2008, 42 (1). https://doi.org/10.1016/j.neuroimage.2008.04.250.

[11] Nolte, G.; Bai, O.; Wheaton, L.; Mari, Z.; Vorbach, S.; Hallett, M. Identifying True Brain Interaction from EEG Data Using the Imaginary Part of Coherency. Clin. Neurophysiol., 2004, 115 (10). https://doi.org/10.1016/j.clinph.2004.04.029.

[12] Pascual-Marqui, R. D.; Lehmann, D.; Koukkou, M.; Kochi, K.; Anderer, P.; Saletu, B.; Tanaka, H.; Hirata, K.; John, E. R.; Prichep, L.; et al. Assessing Interactions in the Brain with Exact Low-Resolution Electromagnetic Tomography. Philos. Trans. R. Soc. A Math. Phys. Eng. Sci., 2011, 369 (1952). https://doi.org/10.1098/rsta.2011.0081.

[13] Marosi, E.; Harmony, T.; Sánchez, L.; Becker, J.; Bernal, J.; Reyes, A.; de León, A. E. D.; Rodríguez, M.; Fernández, T. Maturation of the Coherence of EEG Activity in Normal and Learning-Disabled Children. Electroencephalogr. Clin. Neurophysiol., 1992, 83 (6). https://doi.org/10.1016/0013-4694(92)90070-X.

[14] Fernández, T.; Harmony, T.; Fernández-Bouzas, A.; Silva, J.; Herrera, W.; Santiago-Rodríguez, E.; Sánchez, L. Sources of EEG Activity in Learning Disabled Children. Clin. EEG Electroencephalogr., 2002.

[15] Harmony, T.; Hinojosa, G.; Marosi, E.; Becker, J.; Rodriguez, M.; Reyes, A.; Rocha, C. Correlation between Eeg Spectral Parameters and an Educational Evaluation. Int. J. Neurosci., 1990, 54 (1-2). https://doi.org/10.3109/00207459008986630.

[16] Jäncke, L.; Alahmadi, N. Resting State EEG in Children with Learning Disabilities. Clin. EEG Neurosci., 2016, 47 (1), 24-36. https://doi.org/10.1177/1550059415612622.

[17] John, E. R.; Prichep, L.; Ahn, H.; Easton, P.; Fridman, J.; Kaye, H. Neurometric Evaluation of Cognitive Dysfunctions and Neurological Disorders in Children. Progress in Neurobiology. 1983. https://doi.org/10.1016/0301-0082(83)90014-X.

[18] Byring, R. F.; Salmi, T. K.; Sainio, K. O.; Örn, H. P. EEG in Children with Spelling Disabilities. Electroencephalogr. Clin. Neurophysiol., 1991, 79 (4). https://doi.org/10.1016/0013-4694(91)90119-O. 
[19] Chabot, R. J.; di Michele, F.; Prichep, L.; John, E. R. The Clinical Role of Computerized EEG in the Evaluation and Treatment of Learning and Attention Disorders in Children and Adolescents. J. Neuropsychiatry Clin. Neurosci., 2001, 13 (2), 171-186. https://doi.org/10.1176/appi.neuropsych.13.2.171.

[20] Fonseca, L. C.; Tedrus, G. M. A. S.; Chiodi, M. G.; Cerqueira, J. N.; Tonelotto, J. M. F. Quantitative EEG in Children with Learning Disabilities: Analysis of Band Power. Arq. Neuropsiquiatr., 2006. https://doi.org/10.1590/S0004-282X2006000300005.

[21] Sakkalis, V. Review of Advanced Techniques for the Estimation of Brain Connectivity Measured with EEG/MEG. Comput. Biol. Med., 2011, 41 (12). https://doi.org/10.1016/j.compbiomed.2011.06.020.

[22] Greenblatt, R. E.; Pflieger, M. E.; Ossadtchi, A. E. Connectivity Measures Applied to Human Brain Electrophysiological Data. Journal of Neuroscience Methods. 2012. https://doi.org/10.1016/j.jneumeth.2012.02.025.

[23] Carson, A. M.; Salowitz, N. M. G.; Scheidt, R. A.; Dolan, B. K.; Van Hecke, A. V. Electroencephalogram Coherence in Children with and without Autism Spectrum Disorders: Decreased Interhemispheric Connectivity in Autism. Autism Res., 2014, 7 (3). https://doi.org/10.1002/aur.1367.

[24] Clarke, A. R.; Barry, R. J.; Heaven, P. C. L.; McCarthy, R.; Selikowitz, M.; Byrne, M. K. EEG Coherence in Adults with Attention-Deficit/Hyperactivity Disorder. Int. J. Psychophysiol., 2008, $67 \quad$ (1). https://doi.org/10.1016/j.ijpsycho.2007.10.001.

[25] Duffy, F. H.; Shankardass, A.; McAnulty, G. B.; Als, H. The Relationship of Asperger's Syndrome to Autism: A Preliminary EEG Coherence Study. BMC Med., 2013, 11 (1). https://doi.org/10.1186/1741-7015-11-175.

[26] Srinivasan, R.; Winter, W. R.; Ding, J.; Nunez, P. L. EEG and MEG Coherence: Measures of Functional Connectivity at Distinct Spatial Scales of Neocortical Dynamics. J. Neurosci. Methods, 2007, 166 (1). https://doi.org/10.1016/j.jneumeth.2007.06.026.

[27] Bosch-Bayard, J.; Girini, K.; Biscay, R. J.; Valdes-Sosa, P.; Evans, A. C.; Chiarenza, G. A. Resting EEG Effective Connectivity at the Sources in Developmental Dysphonetic Dyslexia. Differences with Non-Specific Reading Delay. Int. J. Psychophysiol., 2020, 153. https://doi.org/10.1016/j.ijpsycho.2020.04.021.

[28] Arns, M.; Peters, S.; Breteler, R.; Verhoeven, L. Different Brain Activation Patterns in Dyslexic Children: Evidence from EEG Power and Coherence Patterns for the Double-Deficit Theory of Dyslexia. J. Integr. Neurosci., 2007, 6 (1). https://doi.org/10.1142/S0219635207001404.

[29] Marosi, E.; Harmony, T.; Becker, J.; Reyes, A.; Bernal, J.; Fernández, T.; Rodríguez, M.; Silva, J.; Guerrero, V. Electroencephalographic Coherences Discriminate between Children with Different Pedagogical Evaluation. Int. J. Psychophysiol., 1995, 19 (1). https://doi.org/10.1016/0167-8760(94)00059-N.

[30] Jäncke, L.; Alahmadi, N. Resting State EEG in Children With Learning Disabilities: An Independent Component Analysis Approach. Clin EEG Neurosci, 2016, 47 (1). https://doi.org/10.1177/1550059415612622.

[31] Dhar, M.; Been, P. H.; Minderaa, R. B.; Althaus, M. Reduced Interhemispheric Coherence in Dyslexic Adults. Cortex, 2010, 46 (6). https://doi.org/10.1016/j.cortex.2009.09.006. 
[32] Marosi, E.; Harmony, T.; Reyes, A.; Bernal, J.; Fernández, T.; Guerrero, V.; Rodríguez, M.; Silva, J.; Yáñez, G.; Rodríguez, H. A Follow-up Study of EEG Coherences in Children with Different Pedagogical Evaluations. Int. J. Psychophysiol., 1997, 25 (3). https://doi.org/10.1016/S0167-8760(96)00745-3.

[33] Leisman, G. Coherence of Hemispheric Function in Developmental Dyslexia. In Brain and Cognition; 2002; Vol. 48. https://doi.org/10.1006/brcg.2001.1392.

[34] Sklar, B.; Hanley, J.; Simmons, W. W. An EEG Experiment Aimed toward Identifying Dyslexic Children. Nature, 1972, 240 (5381). https://doi.org/10.1038/240414a0.

[35] Fernández, T.; Herrera, W.; Harmony, T.; Díaz-Comas, L.; Santiago, E.; Sánchez, 1.; Bosch, J.; Fernández-Bouzas, A.; Otero, G.; Ricardo-Garcell, J.; et al. EEG and Behavioral Changes Following Neurofeedback Treatment in Learning Disabled Children. Clin. EEG Neurosci., 2003, 34 (3). https://doi.org/10.1177/155005940303400308.

[36] Fernández, T.; Harmony, T.; Fernández-Bouzas, A.; Díaz-Comas, L.; Prado-Alcalá, R. A.; Valdés-Sosa, P.; Otero, G.; Bosch, J.; Galán, L.; Santiago-Rodríguez, E.; et al. Changes in EEG Current Sources Induced by Neurofeedback in Learning Disabled Children. An Exploratory Study. Appl. Psychophysiol. Biofeedback, 2007, 32 (3), 169-183. https://doi.org/10.1007/s10484-007-9044-8.

[37] Fernández, T.; Bosch-Bayard, J.; Harmony, T.; Caballero, M. I.; Díaz-Comas, L.; Galán, L.; Ricardo-Garcell, J.; Aubert, E.; Otero-Ojeda, G. Neurofeedback in Learning Disabled Children: Visual versus Auditory Reinforcement. Appl. Psychophysiol. Biofeedback, 2016, 41 (1). https://doi.org/10.1007/s10484-015-9309-6.

[38] Becerra, J.; Fernández, T.; Harmony, T.; Caballero, M. I.; Garcia, F.; Fernández-Bouzas, A.; Santiago-Rodriguez, E.; Prado-Alcalá, R. A. Follow-Up Study of Learning-Disabled Children Treated with Neurofeedback or Placebo. Clin. EEG Neurosci., 2006, 37 (3). https://doi.org/10.1177/155005940603700307.

[39] Morken, F.; Helland, T.; Hugdahl, K.; Specht, K. Reading in Dyslexia across Literacy Development: A Longitudinal Study of Effective Connectivity. Neuroimage, 2017, 144. https://doi.org/10.1016/j.neuroimage.2016.09.060.

[40] Association, W. M. World Medical Association Declaration of Helsinki: Ethical Principles for Medical Research Involving Human Subjects. JAMA, 2013, 310 (20), 2191-2194.

[41] Matute, E.; Inozemtseva, O.; Gonzalez, A. L.; Chamorro, Y. La Evaluación Neuropsicológica Infantil (ENI): Historia y Fundamentos Teóricos de Su Validación, Un Acercamiento Práctico a Su Uso y Valor Diagnóstico. Rev. Neuropsicol. Neuropsiquiatría y Neurociencias, 2014. https://doi.org/10.1017/CBO9781107415324.004.

[42] Wechsler, D.; Flanagan, D. P.; TEA Ediciones. Departamento I+D. WISC-IV : Escala de Inteligencia de Wechsler Para Niños-IV; 2007.

[43] Ferrando, L.; Bobes, J.; Gilbert, M.; Soto, M.; Soto, O. MINI. MINI International Neuropsychiatric Interview. Versión en español 5.0.0. DSM-IV https://www.academia.cat/files/425-7297-DOCUMENT/MinientrevistaNeuropsiquatribaInternacional.pdf. 
[44] Sheehan, D. V.; Sheehan, K. H.; Shytle, R. D.; Janavs, J.; Bannon, Y.; Rogers, J. E.; Milo, K. M.; Stock, S. L.; Wilkinson, B. Reliability and Validity of the Mini International Neuropsychiatric Interview for Children and Adolescents (MINI-KID). J. Clin. Psychiatry, 2010, 71 (3). https://doi.org/10.4088/JCP.09m05305whi.

[45] Harmony, T.; Marosi, E.; Díaz de León, A. E.; Becker, J.; Fernández, T. Effect of Sex, Psychosocial Disadvantages and Biological Risk Factors on EEG Maturation. Electroencephalogr. Clin. Neurophysiol., 1990, 75 (6). https://doi.org/10.1016/0013-4694(90)90135-7.

[46] Szava, S.; Valdes, P.; Biscay, R.; Galan, L.; Bosch, J.; Clark, I.; Jimenez, J. C. High Resolution Quantitative EEG Analysis. Brain Topogr., 1994, 6 (3), 211-219. https://doi.org/10.1007/BF01187711.

[47] Valdés, P.; Biscay, R.; Galán, L.; Bosch, J.; Zsava, S.; Virués, T. High Resolution Spectral EEG Norms Topography. Brain Topogr., 1990, 3 (December), 281-282.

[48] Caceres, H. A Global Scale Factor in Brain Topography A Global Scale Factor in Brain Topography. 1994, No. July. https://doi.org/10.3109/00207459408986009.

[49] Murias, M.; Swanson, J. M.; Srinivasan, R. Functional Connectivity of Frontal Cortex in Healthy and Adhd Children Reflected in EEG Coherence. Cereb. Cortex, 2007, 17 (8). https://doi.org/10.1093/cercor/bhl089.

[50] Thatcher, R. W.; North, D.; Biver, C. EEG and Intelligence: Relations between EEG Coherence, EEG Phase Delay and Power. Clin. Neurophysiol., 2005, 116 (9). https://doi.org/10.1016/j.clinph.2005.04.026.

[51] Chabot, R. J.; Serfontein, G. Quantitative Electroencephalographic Profiles of Children with Attention Deficit Disorder. Biol. Psychiatry, 1996, 40 (10). https://doi.org/10.1016/0006-3223(95)00576-5.

[52] Barry, R. J.; Clarke, A. R.; McCarthy, R.; Selikowitz, M. EEG Coherence in Attention-Deficit/Hyperactivity Disorder: A Comparative Study of Two DSM-IV Types. Clin. Neurophysiol., 2002, 113 (4). https://doi.org/10.1016/S1388-2457(02)00036-6.

[53] Clarke, A. R.; Barry, R. J.; McCarthy, R.; Selikowitz, M.; Johnstone, S. J.; Abbott, I.; Croft, R. J.; Magee, C. A.; Hsu, C. I.; Lawrence, C. A. Effects of Methylphenidate on EEG Coherence in Attention-Deficit/ Hyperactivity Disorder. Int. J. Psychophysiol., 2005, 58 (1). https://doi.org/10.1016/j.ijpsycho.2005.03.004.

[54] Weiss, S.; Mueller, H. M. The Contribution of EEG Coherence to the Investigation of Language. Brain and Language. 2003. https://doi.org/10.1016/S0093-934X(03)00067-1.

[55] Galaburda, A. M.; Sherman, G. F.; Rosen, G. D.; Aboitiz, F.; Geschwind, N. Developmental Dyslexia: Four Consecutive Patients with Cortical Anomalies. Ann. Neurol., 1985, 18 (2). https://doi.org/10.1002/ana.410180210.

[56] Martin, A.; Schurz, M.; Kronbichler, M.; Richlan, F. Reading in the Brain of Children and Adults: A Meta-Analysis of 40 Functional Magnetic Resonance Imaging Studies. Hum. Brain Mapp., 2015,36 (5). https://doi.org/10.1002/hbm.22749. 